\title{
DEGRADAÇÃO DOS MATERIAIS EM AMBIENTES DO PRÉ- SAL: UMA ABORDAGEM TECNOLÓGICA*
}

Fábio de Oliveira Braga ${ }^{1}$ Sérgio Neves Monteiro ${ }^{2}$ Adriana da Cunha Rocha ${ }^{3}$

\section{Resumo}

A descoberta de óleo e gás no Pré-Sal transformou o Brasil em um dos grandes possuidores de reservas de petróleo no mundo e aumentou as expectativas de produção em águas profundas. Apesar da euforia, alguns desafios têm o potencial de inviabilizar a exploração do Pré-Sal: a complexa logística offshore, as dificuldades de acesso ao reservatório, as condições físico-químicas do poço e de subsuperfície. As condições impostas pelo ambiente são relevantes, devido aos altos teores de contaminantes, alta temperatura e pressão, que exigem materiais de alto desempenho. O objetivo deste trabalho é revisar os avanços que podem contribuir para superar as condições físico-químicas do Pré-Sal. Verificou-se que a solução deste problema pode envolver três tipos de abordagem: O desenvolvimento de ligas resistentes à corrosão (LRC), a adição de agentes modificadores do meio corrosivo (inibidores) e modificações no projeto dos equipamentos. Espera-se, portanto, que a utilização conjunta das diferentes abordagens proporcione a viabilidade de longo prazo da produção no Pré-Sal.

Palavras-chave: Óleo e gás; Ligas resistentes à corrosão; Pré-Sal.

\section{DEGRADATION OF MATERIALS IN THE PRE-SALT LAYER ENVIRONMENT: A TECHNOLOGICAL APPROACH}

\begin{abstract}
The discovery of oil and gas in the Pre-Salt made Brazil one of the biggest owners of petroleum reservoir in the world and increased the expectations of production in deep waters. Despite the excitement, there are some challenges that have the potential to disable the exploration of Pre-Salt: the complex offshore logistics, the difficulties of accessing the reservoir, the physicochemical conditions of the well and subsurface. The conditions imposed by the environment are relevant, due to the high content of contaminants, high temperature and pressure, that requires high performance materials. The objective of this work is to revise the advances which can contribute to overcome the extreme physicochemical conditions of the Pre-Salt. It has been verified that the solution to this problem might involve three types of approach: The development of corrosion resistant alloys (CRA), the addition of modifiers of the corrosion medium (inhibitors) and modifications in the project of the equipment. Therefore, it is expected that the union of the three approaches allows the long-term production in the Pre-salt.
\end{abstract}

Keywords: Oil and gas; Corrosion resistant alloys; Pre-Salt.

1 Engenheiro Metalurgista, M.Sc., Especialista em Engenharia de Inspeção e Manutenção na Indústria do Petróleo, Universidade Católica de Petrópolis. fabio_obraga@yahoo.com.br.

2 Engenheiro Metalurgista, Ph.D., Professor Titular, Seção de Engenharia Mecânica e de Materiais, Instituto Militar de Engenharia, Rio de Janeiro, RJ, Brasil.

3 Engenheira Metalurgista e de Materiais, D.Sc., Professora Adjunta, Programa de Engenharia Metalúrgica e de Materiais, COPPE/UFRJ, Rio de Janeiro, RJ, Brasil. 


\section{INTRODUÇÃO}

A descoberta de grandes quantidades de óleo e gás na camada oceânica conhecida como Pré-Sal tornou o Brasil um dos grandes possuidores de reservas de petróleo no mundo, aumentando as expectativas de produção em águas ultra-profundas. $O$ termo Pré-Sal refere-se a um conjunto de rochas localizadas abaixo de uma espessa camada de sal que têm grande potencial para armazenar petróleo. São reservatórios de difícil acesso, chegando a $\sim 7.000$ metros de profundidade, sendo $\sim 2.000 \mathrm{~m}$ de lâminas d'água e $\sim 5.000 \mathrm{~m}$ de camadas de rochas e sal. São áreas gigantescas, que chegam a cobrir uma área de aproximadamente $150.000 \mathrm{~km}^{2}{ }^{1}$.

A exploração destas reservas poderá tornar o Brasil no $4^{\circ}$ maior produtor de petróleo do mundo até $2030^{2}$. Neste caso, serão necessários altos investimentos, bem como adaptações de logística e tecnologia.

Apesar da euforia, existem alguns desafios que têm o potencial de inviabilizar a exploração do Pré-Sal ${ }^{3-5}$ : (i) As condições severas, como alta pressão e temperatura no poço, e o ambiente corrosivo, devido aos altos teores de gás carbônico $\left(\mathrm{CO}_{2}\right)$ e gás sulfídrico $\left(\mathrm{H}_{2} \mathrm{~S}\right)$ no fluido produzido ${ }^{6-11}$; (ii) A grande lâmina d'água (>2000 m) e a espessa camada de sal $(\sim 2000 \mathrm{~m})$, que dificultam a interpretação dos dados geofísicos e o acesso dos equipamentos ao reservatório ${ }^{4}$; (iii) $\mathrm{O}$ fato de as reservas estarem localizadas a cerca de $300 \mathrm{~km}$ da costa, transformando o simples transporte de pessoas e materiais em um desafio logístico ${ }^{10-11}$.

Alguns dos problemas mais complexos da exploração do Pré-Sal envolvem as condições físico-químicas severas às quais os equipamentos estarão submetidos e a resistência à corrosão dos materiais selecionados é fator de grande importância ${ }^{8,12}$. Dutos, conexões como manifolds, árvores de natal molhadas (ANMs) e linhas de ancoragem, são alguns dos equipamentos de alto custo e de grande responsabilidade que poderão apresentar funcionamento inadequado ou até falhar prematuramente, caso o material selecionado não for adequado ou não forem tomadas medidas de proteção anticorrosiva necessárias.

Entende-se por corrosão a deterioração dos materiais metálicos por mecanismo químico ou eletroquímico, de forma que suas propriedades sejam degradadas e, consequentemente, o funcionamento dos equipamentos pode ser comprometido ${ }^{12}$. Estes fenômenos podem gerar muitas perdas econômicas, bem como afetar a segurança do homem e do meio ambiente. As perdas por corrosão no mundo somam a incrível quantia 2,2 trilhões de dólares por ano, ou seja, cerca de $3 \%$ de tudo que o mundo produz anualmente, como informa a World Corrosion Organization ${ }^{13}$. Por este motivo, as tecnologias de prevenção da corrosão, que minimizem a taxa de falhas e seus custos, precisam ser conhecidas pelas empresas e pela sociedade, sendo necessário um estudo que englobe os mais recentes desenvolvimentos em proteção contra a corrosão, particularmente para área submarina do Pré-Sal. Tendo em vista a importância da corrosão na indústria do petróleo, muitos trabalhos têm sido publicados, buscando solucionar problemas específicos de equipamentos industriais que trabalham em ambiente salino ${ }^{7-9,14-18}$. Assim, o objetivo deste trabalho é rever os avanços recentes que podem contribuir para superar as condições físico-químicas extremas do Pré-Sal, diminuindo-se as perdas por corrosão. 


\section{ABORDAGEM}

Neste trabalho buscou-se verificar o que há de relevante nos artigos e publicações especializadas recentes, acerca dos problemas tecnológicos relacionados às condições físico-químicas dos reservatórios e poços do Pré-Sal. Buscou-se identificar algumas tecnologias que poderão contribuir para a superação dos desafios. Sendo assim, o trabalho foi dividido em duas partes. Na primeira, serão discutidos os agentes físico-químicos responsáveis pelas dificuldades operacionais da exploração de óleo e gás em águas profundas, especialmente nas áreas do PréSal. Na segunda parte serão discutidas algumas tecnologias recentes que os pesquisadores têm desenvolvido no mundo, que podem ter impacto positivo na transposição dos desafios do Pré-Sal. As abordagens para solução foram divididas em três grandes grupos, como já classifica a literatura clássica ${ }^{12}$ : modificação do material; modificação do meio corrosivo e modificações de projeto dos equipamentos.

\section{DIFICULDADES E SOLUÇÕES}

\subsection{Agentes físico-químicos}

Fatores como velocidade dos ventos, altura das ondas, direções das correntes marinhas, ocorrência de tempestades, altas pressões hidrostáticas (grande coluna d'água), baixas temperaturas no fundo do mar, altas temperaturas e pressões nos poços, são algumas características desafiadoras das regiões do Pré-Sal ${ }^{3,7}$. Sabe-se os fluidos produzidos no Pré-Sal têm algumas peculiaridades em relação aos das outras locações, como a alta razão gás-óleo, bem como a forte presença de $\mathrm{CO}_{2} \mathrm{e}$ $\mathrm{H}_{2} \mathrm{~S}$ nos hidrocarbonetos. Estas são substâncias conhecidas por acelerar o processo de corrosão nos metais, tornando o meio corrosivo mais agressivo ${ }^{4,8,12,14}$. Este problema é agravado pela dificuldade de monitoração das taxas de corrosão e identificação das falhas nos materiais.

A seleção dos materiais para trabalhar em meios corrosivos desconhecidos é inerentemente uma tarefa complexa, pois é necessária a coleta de amostras dos fluidos, a reprodução do meio em laboratório e o teste das novas ligas ${ }^{19-23}$. No caso do Pré-Sal, esta tarefa torna-se mais difícil, devido às grandes dimensões geográficas, e às grandes profundidades, às quais estão associadas as diferentes pressões, temperaturas e tipos de agentes corrosivos. Neste caso, a composição dos fluidos nos reservatórios e as condições operacionais dos equipamentos podem variar muito, e o processo de seleção dos materiais pode ser um grande desafio.

Um complicador da exploração em águas profundas é o constante risco de formação de hidratos ${ }^{3,4,7}$. No reservatório, os hidrocarbonetos são armazenados a altas temperaturas e pressões, e na produção do petróleo, estes fluidos são conduzidos por dutos até a Unidade de Produção, sujeitos a perdas de temperatura. No Pré-Sal, a temperatura do leito marinho é baixa, e se há excesso de troca térmica entre o fluido produzido e o ambiente, pode haver depósito de parafinas, hidratos e asfalteno, que podem restringir ou impedir a passagem do fluido.

Outras condições típicas do Pré-Sal são as grandes lâminas d'água ( $2.000 \mathrm{~m})$ e as ondas $40 \%$ mais altas que nos campos do Pós-Sal da Bacia de Campos, que resultam da maior distância da costa. Estas características tornam imperativo um sistema de ancoragem mais robusto, que mantenha as Unidades de Produção com o passeio adequado ${ }^{3,4}$. 


\subsection{Novas tecnologias}

\subsubsection{Modificação do material}

A modificação do material consiste na alteração das características inerentes ao material, com o objetivo de melhorar a resistência à corrosão. As características que podem ser controladas são a composição química (elementos de liga e nível de impurezas) e a microestrutura (processos de obtenção do material, tratamentos térmicos e/ou mecânicos) ${ }^{24}$. A seleção de um material distinto para uma dada aplicação, bem como a modificação superficial de um material também serão considerados nesta categoria. Algumas formas de modificação da superfície incluem tratamentos térmicos, mecânicos, químicos e/ou aplicação de revestimentos ${ }^{12}$. Alguns exemplos de revestimentos são a deposição eletroquímica ou metalúrgica de um metal (como por fusão ou colaminação a quente), a aplicação de materiais orgânicos (tintas, resinas ou polímeros em geral) e inorgânicos (esmaltes, cimentos). Em geral, a aplicação de revestimentos permite que apenas as partes mais críticas de uma estrutura submersa, como a parte interna de um riser de produção, sejam feitas de um material mais nobre, e, portanto, mais caro $8,12,21,23$.

A seleção de uma liga resistente à corrosão (LRC) para equipamentos submersos pode ser um processo complexo, especialmente em condições severas como o PréSal. Buscando soluções, vários pesquisadores têm se dedicado a levantar informações e criar metodologias para seleção de ligas para ambientes corrosivos $^{8,12,20-22,24}$. Uma abordagem comum e eficaz para a escolha de uma LRC é fazer uma pré-seleção de conjunto de ligas mais adequadas com base em dados da literatura, levando-se em conta também o aspecto econômico, realizar testes preliminares, e, finalmente, realizar testes mais detalhados em um ambiente que simula o real, para a escolha final ${ }^{19-20}$. O processo de seleção de materiais deve levar em conta além do desempenho (propriedades mecânicas e resistência à corrosão), o peso, custo dos materiais, ainda que estas sejam, em muitos casos, demandas concorrentes. A facilidade de união por solda (soldabilidade) e disponibilidade no mercado também são outros fatores relevantes ${ }^{3,24}$.

Para equipamentos submetidos a altas pressões (poços ou linhas submersas), a alta pressão parcial do $\mathrm{CO}_{2}$ e/ou a presença de $\mathrm{H}_{2} \mathrm{~S}$ indica a necessidade do uso de ligas especiais ${ }^{3,21,25-27}$. Algumas ligas já utilizadas na indústria de óleo e gás são os aços 13Cr (supermartensítico UNSS41425), 2507 (duplex S32750) e ligas de Ni 2535 (N08535) e 29 (N08029), para ambientes com $\mathrm{H}_{2} \mathrm{~S}$ e cloretos ${ }^{26}$. Outras ligas que foram testadas recentemente são os hastelloy C-22 (N10276), C-22HS (N07022), C-276 (N10276) e C-2000 (N06200), para ambientes ricos em $\mathrm{H}_{2} \mathrm{~S}^{25}$.

A Tabela 1 mostra algumas das LRC comumente utilizadas submersas em ambientes salinos offshore ${ }^{3}$. Além dos teores dos principais elementos de liga, são mostrados o limite de escoamento $\left(\sigma_{e}\right)$ e um parâmetro que quantifica a resistência à corrosão por pites (PREN, do inglês Pit Resistance Equivalent Number) ${ }^{3,8,21}$. Em virtude da alta corrosividade do meio salino, tradicionalmente, os aços inoxidáveis têm sido os preferidos para aplicações de longo prazo. Os aços de microestrutura duplex (como os aços UNS 32205 e 32750) foram concebidos para conciliar as características de resistência mecânica e à corrosão, que costumam ser antagônicas no caso dos aços inoxidáveis. 
Tabela 1. Ligas resistentes à corrosão e algumas de suas propriedades

\begin{tabular}{cccccccc}
\hline \multirow{2}{*}{ NOME } & \multirow{2}{*}{ TIPO } & \multicolumn{3}{c}{ COMPOSIÇÃO QUÍMICA } & \multicolumn{2}{c}{$\boldsymbol{\sigma}_{\mathbf{e}}$} & \multirow{2}{*}{ PREN } \\
\cline { 3 - 6 } & & $\mathbf{C r}$ & $\mathbf{N i}$ & $\mathbf{M o}$ & $\mathbf{C u}$ & $(\mathbf{M P a})$ & \\
\hline $13 \mathrm{Cr}$ & Supermartensítico & 13 & - & - & - & 550 & 13 \\
\hline S13Cr & Supermartensítico & 13 & 5 & 2 & - & 550 & 20 \\
\hline 316 & Austenítico SS & 17 & 12 & 2,2 & - & 205 & 24 \\
\hline 317 & Austenítico SS & 18 & 15 & 4,5 & - & 205 & 29 \\
\hline 32205 & Duplex SS & 22 & 5,6 & 2,8 & - & 450 & 34 \\
\hline 32750 & Super-duplex SS & 25 & 7 & 3,5 & - & 550 & 41 \\
\hline 904 & Superaustenítico & 20 & 25 & 4,2 & 1,5 & 220 & 36 \\
\hline 31266 & Superaustenítico & 25 & 22 & 5,8 & 1,5 & 220 & 55 \\
\hline 825 & Liga de Níquel & 22 & 42 & 3 & 2,5 & 440 & 32 \\
\hline 625 & Liga de Níquel & 21 & 70 & 9 & - & 517 & 51 \\
\hline C276 & Liga de Níquel & 16 & 68 & 16 & - & 355 & 68 \\
\hline
\end{tabular}

Fonte: BELTRÃO et al. ${ }^{3}$

Beltrão et al. ${ }^{3}$, listaram os materiais mais comuns para as aplicações específicas offshore. Eles dividiram nas categorias "equipamentos de poço", "linhas submersas" e "equipamentos topside".

- Equipamentos de poço: Nos componentes em contato com o fluido produzido, os aços superduplex 32750 são os utilizados (alta corrosividade). Se o pH for relativamente alto, os aços $13 \mathrm{Cr}-5 \mathrm{Ni}-2 \mathrm{Mo}$ podem ser considerados. Aço carbono comum pode ser utilizado se não há contato com o fluido produzido. Já as árvores de natal molhadas (ANM) costumam ser cladeadas com inconel 625, material custoso e de baixa disponibilidade. $\mathrm{O}$ aço $13 \mathrm{Cr}$, por outro lado, possui menor custo e maior disponibilidade no mercado, mas sua aplicação é restrita a teores de cloreto menores que 50.000 ppm, o que inviabilizaria o seu uso no Pré-Sal. Apesar disso, o estudo do $13 \mathrm{Cr}$ foi retomado posteriormente para utilização no Pré-Sal ${ }^{19}$, sendo descoberto que este pode substituir o superduplex nas colunas de produção e de revestimento, pois, apesar do alto teor de $\mathrm{CO}_{2}$ do meio, a interação entre as rochas do reservatório e a água da formação torna o ambiente menos severo.

Outro aço, o 13Cr-5Ni-2Mo, por sua vez, foi estudado para uso em ambiente aquoso salino (cloretos) com $\mathrm{CO}_{2}$ e H2S. Foi identificado que o $\mathrm{pH}$ deveria ser superior a 4 para não haver risco de falha por corrosão. Se não for possível o uso do $13 \mathrm{Cr}-5 \mathrm{Ni}-$ $2 \mathrm{Mo}$, o aço superduplex 25Cr-7Ni-3Mo pode ser considerado 3 .

- Linhas submersas: Linhas flexíveis são uma opção, mas a corrosão-fadiga da armadura do riser deve ser considerada. A injeção de inibidor de corrosão pode ser necessária, para evitar a deterioração da armadura pelo $\mathrm{H}_{2} \mathrm{~S}$. Armaduras externas em fibra de carbono também têm sido estudadas, para melhor resistência à fadiga $e$ à corrosão ${ }^{28}$. A permeação do $\mathrm{CO}_{2}$ pela camada polimérica do riser pode ser um problema. Linhas rígidas são a outra opção, mas é necessário cladeamento com inconel 625 em regiões mais críticas. Para as flowlines (como são chamadas as linhas em contato com o solo marinho), aços superduplex são a melhor opção. Cuidados devem ser tomados para prevenir trincamento pelo hidrogênio, devido à interação com a proteção catódica.

- Equipamentos topside: Devido à menor pressão, e à menor pressão parcial do $\mathrm{CO}_{2}$, é possível o uso de aço carbono comum. Em casos mais críticos pode ser usado o duplex 22Cr. Em vasos de pressão, cladeamento com Inconel 625 ou revestimento epoxy podem ser utilizados.

Popoola et al. ${ }^{8}$, listaram outras ligas especiais utilizadas na indústria do petróleo, que são utilizados para resolver problemas de corrosão: $28 \mathrm{Cr}$ inoxidável, LDX 2101 
254SMO e 654 SMO, e as liga de níquel 825, 2550 e C276, que também podem ser consideradas para aplicação no Pré-Sal. Mais recentemente, foi desenvolvido um aço para revestir as paredes de poços e colunas de produção do Pré-Sal, denominado $\mathrm{Cr} 17^{19}$. Apesar de custoso e de menor disponibilidade, após mais pesquisas é esperado que exista viabilidade de aplicação.

O uso de revestimentos também pode ser uma boa alternativa para diminuir os custos com materiais, protegendo contra a corrosão somente as regiões críticas, como as de contato com o fluido corrosivo. Popoola et al. ${ }^{8}$, revisaram o uso de revestimentos protetores para mitigação de problemas de corrosão na indústria de óleo e gás. Segundo os autores, os revestimentos mais utilizados como proteção externa de equipamentos são os epóxi unidos por fusão (FBE, do inglês fusionbonded epoxy) e as poliolefinas tricamada (3LPO, do inglês 3-layer polyolefin). As poliolefinas mais utilizadas são o polietileno e o polipropileno. Outras soluções em revestimentos incluem: Sistema de Revestimento Compósito de Alto Desempenho (HPCC, do inglês High Performance Composite Coating System), Tecnologia de Aplicação de Baixa Temperatura para Revestimento por Pó em Aços de Alta Resistência e a Tecnologia Thermotite ${ }^{\circledR}$ de Revestimento para Garantia de Escoamento ${ }^{8}$. Outro caso já mencionado de revestimento são os tubos cladeados. Uma nova tecnologia de aplicação destes revestimentos para a indústria do óleo e gás é o cladeamento por explosão ${ }^{29}$.

O acompanhamento das mudanças nos materiais ao longo do tempo pode ser importante para evitar falhas catastróficas devido à corrosão. Diversos métodos de inspeção de estruturas submersas foram revisados por Liu e Kleiner ${ }^{30}$. Foram analisados métodos visuais, eletromagnéticos, acústicos, ultrassônicos, radiográficos e termográficos. Segundo os autores, alguns dos métodos são custosos, e seu uso só se justifica em seções de tubos de grande importância. Como os poços do Pré-Sal receberão equipamentos de ponta, e, em alguns casos, tubos que desempenham funções de grande responsabilidade, a utilização de métodos sofisticados poderá ser justificável e até essencial para evitar falhas. Ainda segundo os autores ${ }^{23}$, apesar do alto custo, com o aumento do uso de várias destas tecnologias, a tendência é a diminuição do seu custo.

\subsubsection{Modificação do meio corrosivo}

Nesta categoria estão presentes processos de adição de inibidores de corrosão, bem como de remoção de íons e/ou outras substâncias nocivas aos metais, como o que ocorre na desaeração da água, dessalinização, neutralização do meio, entre outras. Neste trabalho, será tratado somente o caso da adição dos inibidores de corrosão, por ser uma alternativa muito utilizada na produção offshore do petróleo. Os inibidores de corrosão consistem em substâncias ou misturas que, quando presentes em concentrações adequadas no meio corrosivo, reduzem ou eliminam a corrosão ${ }^{12,24,31}$. Eles protegem a superfície metálica mesclando-se a ela ou reagindo com as impurezas do ambiente que causariam a corrosão. Os mecanismos de atuação variam: Os inibidores podem diminuir a velocidade dos processos anódicos ou catódicos, bloqueando os sítios ativos na superfície metálica. Podem agir aumentando o potencial da superfície metálica, de forma que o metal sofre o fenômeno de passivação, no qual são formados óxidos protetores. Podem ainda formar compostos na superfície metálica diminuem o processo de corrosão $0^{8,31}$. Alguns inibidores utilizados na indústria do petróleo são mostrados na Tabela 2, juntamente com algumas de suas principais aplicações ${ }^{8}$. 
Tabela 2. Inibidores mais comuns e sua aplicação na indústria de óleo e gás

\section{INIBIDORES}

3-fenil-2-propin-1-ol

\section{APLICAÇÕES EM ÓLEO E GÁS}

Tubos de campo API J55 em soluções de $\mathrm{HCl}$ em diferentes condições

Hidrazidas e tiossemicarbazidas de
ácidos gordos com 11,12 e 18 átomos
de carbono

Mistura de cetonas, quinolínio, sais e ácido fórmico

2-undecano-5-mercapto-1-oxi-3,4-diazol

2-heptadeceno-5-mercapto-1-oxi-3,4diazol

2-deceno-5-mercapto-1-oxi-3,4-diazol

Dibenzilideno acetona

Di-N-dimetilaminobenzilideno

Metoxi fenol e nonil fenol

$\mathrm{N}$-(5,6-difenil-4,5-dihidro-[1,2,4]-triazin-3-

il)-guanidina

6-benzilaminopurina

Mistura de magnetita sintética e gluconato ferroso

Rosin amida imidazolina
Aço macio e aço N80 para poços de petróleo em soluções com $15 \%$ de ácido hidroclórico em ebulição

Instalações tubulares de campo de petróleo em temperaturas tão altas quanto $200^{\circ} \mathrm{C}$ em hidroclórico

Aço macio em $15 \% \mathrm{HCl}$ em $105 \pm 2^{\circ} \mathrm{C}$ e aço $\mathrm{N} 80 \mathrm{em} 15 \% \mathrm{HCl}$ contendo 5.000 ppm de 2-undecano-5-mercapto-1-oxi3,4-diazol

Aço macio e aço $\mathrm{N} 80 \mathrm{em} \mathrm{HCl}$

Aços $\mathrm{N} 80$ em $15 \% \mathrm{HCl}$ em diferentes poríodos de exposição e temperaturas

Aço macio em $1 \mathrm{M}$ de ácido hidroclórico e $0,5 \mathrm{M}$ de ácido sulfúrico

Aços laminados à frio em 1 a $7 \mathrm{M}$ de $\mathrm{H}_{2} \mathrm{SO}_{4}$ de 25 a $50^{\circ} \mathrm{C}$

Aço N80 para poços de petróleo em 50

$\mathrm{mg} / \mathrm{L}$ de concentração de sulfetos em vários $\mathrm{pH}(5,5$ a 11,5$)$ e em altas temperaturas e pressões

Aços N80 e P110 em água de formação saturada de $\mathrm{CO}_{2}$

Fonte: POPOOLA et al. ${ }^{8}$.

Muitos dos inibidores comerciais utilizados na indústria de óleo e gás são misturas que contém surfactantes, desemulsificantes, removedores de oxigênio, além do agente inibidor. A maioria dos inibidores de corrosão utilizados atualmente contém moléculas nitrogenadas, e podem ser classificados em grupos como amidas/imidazolinas, sais de moléculas nitrogenadas com ácidos carboxílicos, aminas polialquiladas, amidas, imidazolinas e heterociclos de nitrogênio. Inibidores não nitrogenados são menos utilizados ${ }^{31}$. As imidazolinas são os inibidores mais utilizados em ambientes ricos em $\mathrm{CO}_{2}{ }^{32}$.

Miksic, Kharshan e Furman ${ }^{33}$, avaliaram diversos tipos de inibidores de corrosão para a indústria do petróleo em diferentes ambientes. Aminas de cadeia longa, amidas gordas, ácidos gordos, e seus sais. Foram testadas diferentes concentrações em meio de hidrocarboneto contendo $\mathrm{CO}_{2}$ e $\mathrm{H}_{2} \mathrm{~S}$. Os produtos forneceram alta proteção do aço nos diferentes tipos de ataque corrosivo.

Alguns novos inibidores têm sido desenvolvidos com a finalidade de atender demandas de desempenho, bem como demandas ambientais. Yang et al. ${ }^{34}, 2016$, estudaram um novo inibidor denominado imidazolina Gemini, que apresentou bom desempenho em ambientes ricos em $\mathrm{CO}_{2}$, em menores concentrações que as imidazolinas comuns, reduzindo o custo e o impacto ambiental. A sua mistura com ácidos gordos também resultou em melhor resultado que as imidazolinas comuns. Mazumder, Al-Muallen e Ali ${ }^{35}, 2015$, estudaram três derivados de imidazolinas, 
dietilenotetramina e tetraetilenopentamina, possuindo diferentes grupos laterais na cadeia, para inibição da corrosão em aço carbono em uma solução $0,5 \mathrm{M}$ de $\mathrm{NaCl} \operatorname{com} \mathrm{CO}_{2}$. Mostrou-se que o poder de inibição aumentou à medida que o comprimento da ramificação aumentou. Mackenzie et al. ${ }^{36}, 2012$, testaram uma metodologia de medir e controlar a concentração ótima de inibidores formadores de películas, pela formação de micelas na fase aquosa. Técnicas analíticas comprovaram a eficácia do método, que viabiliza o desenvolvimento de sensores portáteis para controle da concentração de inibidor. Os exemplos anteriores mostram que a tecnologia de inibidores tem avançado, e que a sua evolução pode gerar produtos que auxiliem superar os altos teores de $\mathrm{CO}_{2}$ e $\mathrm{H}_{2} \mathrm{~S}$ do PréSal.

\subsubsection{Modificação de projeto dos equipamentos}

Nesta categoria podem ser englobadas as alterações nos equipamentos que envolvem modificação do projeto da estrutura, como modificação geométrica, modos junção (rebitagem e colagem), o contato entre metais ou sua ausência e o uso proteção catódica. Gentil ${ }^{12}$ apresenta algumas medidas que devem ser colocadas em prática em qualquer projeto, com a finalidade de evitar a corrosão: (i) Utilizar soldas bem acabadas e contínuas (evita corrosão em frestas); (ii) não formar ângulos fechados e estrangulamentos desnecessários em tubulações (evita ação erosiva no meio); (iii) evitar contatos diretos de materiais metálicos de potenciais eletroquímicos diferentes, ou utilizar isolantes entre eles; (iv) facilitar a completa drenagem de líquidos estagnados. Essas e outras medidas fazem parte das boas práticas de projeto para diminuir o efeito da corrosão. Devem ser aplicadas em conjunto a uma correta seleção das ligas que irão trabalhar e, se for o caso, com a correta seleção de um inibidor e/ou revestimento.

Outra alteração que pode ser necessária é a utilização de proteção catódica ou proteção anódica. A proteção catódica é um método que reduz a corrosão pela minimização da diferença de potencial entre anodo e catodo ${ }^{8,12}$, aplicando-se uma corrente elétrica de uma fonte externa na estrutura a ser protegida. Apesar de poder ser utilizada em estruturas completamente nuas, sua aplicação torna-se bastante econômica quando as superfícies a proteger são completamente revestidas. A proteção catódica só pode ser utilizada no caso de estruturas enterradas ou submersas, pois há a necessidade de o eletrólito ser contínuo. Desenvolvimentos em medição remota dos potenciais de eletrodo estão entre os avanços recentes ${ }^{36}$.

A proteção anódica, por sua vez, é baseada na aplicação de um potencial anódico na superfície do metal com a finalidade de estimular a formação de uma película apassivadora. Ela somente pode ser empregada com sucesso em metais e ligas capazes de formar películas apassivadoras contínuas e aderentes, como o Ti, ligas $\mathrm{Fe}-\mathrm{Cr}$ e $\mathrm{Fe}-\mathrm{Cr}-\mathrm{Ni}$. O seu principal emprego é em meios de alta agressividade, como em tanques de armazenamento de ácidos.

\section{CONCLUSÃO}

O presente trabalho permitiu verificar alguns desenvolvimentos tecnológicos relevantes que podem contribuir na superação das condições extremas do Pré-Sal. Foram identificados os principais agentes físico-químicos de degradação dos materiais nas áreas do Pré-Sal, e algumas abordagens para diminuição das dificuldades operacionais.

Verificou-se que a superação do ambiente corrosivo pode envolver três diferentes abordagens, o desenvolvimento de LRC, a adição de modificadores do meio corrosivo e modificações no projeto dos equipamentos. 
Espera-se, portanto, que a utilização conjunta das diferentes abordagens proporcione a viabilidade de longo prazo da produção no Pré-Sal, através da correta seleção das LRC, do uso de inibidores nos locais e concentrações corretas, e da execução das boas práticas de projeto dos equipamentos.

\section{REFERÊNCIAS}

1 Oxford Analytica. The Impact of pre-salt: A long term perspective. A report prepared for Petrobras. NW Washington: Oxford Analytica; 2010 [acesso em 10 abr 2016].

Disponível em: http://tinyurl.com/zbez4fd.

2 Petrobras [homepage da internet]. Atuação no Pré-Sal [acesso em 22 jul 2015]; Disponível em: http://www.petrobras.com/pt/energia-e-tecnologia/fontes-de-energia/presal/. 2015.

3 Beltrão RLC, Sombra CL, Lage ACVM, Fagundes Netto JR, Henriques CCD. Challenges and new technologies for the development of the pre-salt cluster, Santos Basin, Brazil. One Petro [periódicos na Internet]. 2009 [acesso em 10 abr. 2016]; Disponível em: https://www.onepetro.org/conference-paper/OTC-19880-MS.]

4 Morais JM. Petróleo em Águas Profundas: Uma história tecnológica da Petrobras na exploração e produção offshore. Brasília: IPEA; 2013.

5 Petrobras [homepage da internet]. Tecnologias Pioneiras do Pré-Sal [acesso em 10 abr. 2016]. Disponível em http://presal.hotsitespetrobras.com.br/tecnologiaspioneiras/\#0.

6 Cezar ASP, Pereira AR, Daniel LL, OAZEN EV, Trovoado LC, Almeida MC. Subsea solutions in the pre-salt development projects. One Petro [periódicos da internet]. 2014 [acesso em 10 abr. 2016]. Disponível em: http://tinyurl.com/hzxt49g.

7 Cardoso CABR, Gonçalves MAL, Camargo RMT. Design options for avoiding hydrates in deep offshore production. J. Chem. Eng. Data 2015; 60:330-35.

8 Popoola LT, Grema AS, Latinwo GK, Gutti B, Blogun AS. Corrosion problems during oil and gas production and its mitigation. Int. J. Ind. Chem. 2013; 4:35.

9 Taylor GN, Magalhães AAO, Bustamante FCR, Bonfim ALC. Development and evaluation of a novel, rapid and highly effecient $\mathrm{H}_{2} \mathrm{~S}$ scavenger to meet the challenges of pre-salt offshore applications in Brazil. One Petro [periódicos da internet]. 2015 [acesso em 10 abr. 2016]. Disponível em: http://tinyurl.com/ir2jk6x.

10 Terra AO, Ferreira AS, Nascimento DCO. Os desafios do pré-sal brasileiro: Um estudo de logística do campo de tupi. Anais do XI Simpósio de Excelência em Gestão e Tecnologia; 2014 out. 22-24 [acesso em 02 Set. 2015]. Disponível em: www.aedb.br/seget/arquivos/artigos14/47420570.pdf.

11 Silva VA, Liang DA, Yuba DGT, Rosseto R, Kovalski S. Logistics planning for critical cargos related to hydrogen sulfide removal processes in pre-salt basin fields. One Petro [periódicos da internet]. 2015 [acesso em 10 abr. 2016]. Disponível em: http://tinyurl.com/zjjagzp.

12 Gentil V. Corrosão. 6a ed. Rio de Janeiro: LTC; 2011.

13 Hays GF. Now is the time. Nova York: World Corrosion Organization, 2013 [acesso em 25 set. 2015]. Disponível em: <corrosion.org>.

14 Melchers RE. Long-term corrosion of cast irons and steel in marine and atmospheric environments. Corrosion Science 2013; 68:186-94.

15 Cheng-Hong P, Zheng-Yi L, Xing-Zhao W. Failure analysis of a steel tube joint perforated by corrosion in a well-drilling pipe. Eng. Fail. Anal. 2012; 25:13-28.

16 Le Gac P-Y, Arhant M, Davies P, Muhr A. Fatigue behavior of natural rubber in marine environment: comparison between air and sea water. Mat. Des. 2015; 65:462-67.

17 Mansor NII, Abdullah S, Ariffin AK, Syarif J. A review of the fatigue failure mechanism of metallic materials under a corroded environment. Eng. Fail. Anal. 2014; 42:353-65.

18 Mohd MH, Paik JK. Investigation of the corrosion progress characteristic of offshore subsea oil well tubes. Corrosion Science 2013; 67:130-41. 
19 Joia CJBM. Super-alloys for Pre-Salt [entrevista à homepage da Petrobras]. Petrobras 2015 [acesso em 10 abr. 2016. Disponível em:

http://www.petrobras.com/en/magazine/post/super-alloys-for-pre-salt.htm.

20 Craig BD, Smith L. Corrosion resistant alloys (CRAs) in the oil and gas industry selection guidelines update. $3^{\mathrm{a}} \mathrm{ed}$. Toronto: Nickel Institute, 2011.

21 Silva DM, Souza Jr. HA, Aguiar LAD, Souza AP. Challenges on designing pipelines for the brazilian pre-salt scenarios. Proceedings of the Conference \& Exposition Rio Pipeline. Rio de Janeiro, 2011.

22 JFE Technical Report. Corrosion Resistant High Cr Steel for Oil and Gas Wells. New Products \& Technologies 2013; 18.

23 Henriques CCD, Joia CJB, Guedes F, Baptista IP. Materials selection for Brazilian presalt field. One Petro [periódicos na Internet]. 2012 [acesso em 10 abr. 2016]; Disponível em: http://tinyurl.com/zk88he8.

24 Callister Jr. WD. Ciência e engenharia de materiais: Uma introdução. $7^{a}$ ed. Rio de Janeiro: LTC. 2012.

25 Pike LM, Manning PE, Hibner EL. Severe sour gas testing of a high strength, corrosion resistant Ni-Cr-Mo alloy. One Petro [periódicos na Internet]. 2012 [acesso em 11 abr. 2016]; Disponível em: http://tinyurl.com/hd7nptb.

26 Anderko A, Engelhardt GR, Gui F, Cao L, Sridhar N. Localized corrosion of corrosionresistant alloys in environments containing hydrogen sulfide. One Petro [periódicos na Internet]. 2012 [acesso em 11 abr. 2016]; Disponível em: http://tinyurl.com/hydmzj3.

27 Zin ZM, Ong G, Riyanto L, Jadid M. Fit-for-purpose qualification for corrosion resistant alloy (CRA) tubing slsection in high $\mathrm{CO}_{2}$ field based on field experience and laboratory test. One Petro [periódicos na Internet]. 2012 [acesso em 11 abr. 2016]; Disponível em: http://tinyurl.com/zztnhlv.

28 Hanonge DH, Bernard G, Do AT. Carbon fiber armors applied to pre-salt flexible pipe developments. One Petro [periódicos na Internet]. 2013 [acesso em 10 abr. 2016]; Disponível em: http://tinyurl.com/jlvrro8.

29 Banker JG, Massarelo J, Pauly S. Explosion clad for upstream oil and gas equipment. Proceedings of the International Conference on Advances in Materials and Processing Technologies 2010. Paris: American Institute of Physics, 2010.

30 Liu Z, Kleiner Y. State of the art review of inspection technologies for condition assessment of water pipes. Measurement 2013; 46:1-15.

31 Rahuma MN, Kannan M B. Corrosion in oil and gas industry: a perspective on corrosion inhibitors. J. Material Sci. Eng. 2014; 3(3):1000e110.

32 Mazumder MAJ, Al-Muallen HA, Ali SA. The effect of $\mathrm{N}$-pendants and electron-rich amidine motifs in 2-(p-alkoxyphenil)-2-imidazolines on mild steel corrosion in $\mathrm{CO}_{2}$ saturated 0.5 M NaCl. Corrosion Science 2015; 90:54-68.

33 Miksic BM, Furman AY, Kharshan MA. Effectiveness of the corrosion inhibitors for the petroleum industry under various flow conditions. Proceedings of the Corrosion Conference \& Expo 2009. Houston: NACE International. 09573.

34 Yang J, Gao L, Liu X, Qin W, Yin C, Zhang J. A highly effective corrosion inhibitor by use of Gemini imidazoline. One Petro [periódicos na Internet]. 2016 [acesso em 11 abr. 2016]; Disponível em: http://tinyurl.com/h4fxger.

35 Mackenzie C, Rowley-Williams C, Achour M, Blumer D, Joosten MW, Rowe M. Potential for measurement of corrosion-inhibitor-micelle presence as an indicator of optimum dose. One Petro [periódicos na Internet]. 2016 [acesso em 11 abr. 2016]; Disponível em: http://tinyurl.com/zxpm9xi.

36 Bustamante MA, Baca AJ. System to telemeasure the cathodic protection potentials using fixed reference electrodes in petroleum platforms. One Petro [periódicos na Internet]. 2016 [acesso em 11 abr. 2016]; Disponível em: http://tinyurl.com/pap7zua. 